\title{
Subsequent abdominal surgery after laparoscopic ventral and incisional hernia repair with an expanded polytetrafluoroethylene mesh: a single institution experience with 72 reoperations
}

\author{
E. B. Wassenaar · E. J. P. Schoenmaeckers • \\ J. T. F. J. Raymakers · S. Rakic
}

Received: 30 May 2009 / Accepted: 18 September 2009 / Published online: 6 October 2009

(C) The Author(s) 2009. This article is published with open access at Springerlink.com

\begin{abstract}
Purpose Laparoscopic ventral and incisional hernia repair (LVIHR) carries a risk of adhesion formation and can influence subsequent abdominal operations (SAOs). We performed a retrospective study of findings during reoperations of patients who had previously had an LVIHR by using an expanded polytetrafluoroethylene mesh (DualMesh ${ }^{\circledR} ; \mathrm{WL}$ Gore, Flagstaff, AZ, USA).

Methods The medical records of all 695 patients who had LVIHR at our hospital were reviewed. Patients who underwent SAO for various indications were identified $(n=72)$ and analyzed.

Results Seven LVIHR patients (1\%) had early SAO (within a few days). In six patients $(86 \%)$, removal of the mesh was required. Intra-operatively, in all six of these patients with peritonitis, there were no adhesions against the implant identified. Late SAOs (after more than 1 month) were performed in 65 patients $(9.4 \%)$. Only one patient required acute surgical intervention due to an LVIHR-related adhesion (0.15\%). Laparoscopy was performed in $83 \%$ and laparotomy in $17 \%$ of patients. Adhesions against the implant were present in $83 \%$ of patients; in $65 \%$, the adhesions involved omentum only, and in $18 \%$, they involved the bowel. Adhesiolysis was always easy and caused no inadvertent enterotomies. SAOs were devoid of postoperative complications.

Conclusions In this largest series of reoperations after LVIHR, the majority of patients had mild or moderate adhesions against the implant. The specific observations that: (1) no relaparoscopies had to be converted, (2) no
\end{abstract}

E. B. Wassenaar $(\varangle)$ · E. J. P. Schoenmaeckers ·

J. T. F. J. Raymakers · S. Rakic

Department of Surgery, Twenteborg Hospital,

Postbox 7600, 7600 SZ Almelo, The Netherlands

e-mail: ebwassenaar@me.com inadvertent enterotomies were made during adhesiolysis, and (3) SAOs have practically been devoid of peri- and postoperative complications indicate that SAOs can be safely performed after previous LVIHR with DualMesh.

Keywords Laparoscopic repair · Ventral incisional hernia · Adhesions $\cdot$ Reoperations $\cdot$ Mesh

\section{Introduction}

Classic prosthetic materials used in conventional incisional hernia repair carry a potential to induce severe complications when placed in the abdominal cavity. Complications such as the development of dense adhesions, bowel erosions, and enterocutaneous fistulae have been reported [1-3]. They may also significantly complicate subsequent abdominal surgery [3]. Therefore, the application of these materials in laparoscopic ventral and incisional hernia repair (LVIHR) is, nowadays, generally avoided. New prosthetic materials that present higher biocompatibility trigger less foreign-body reaction, and are more suitable for placement within the peritoneal cavity, provided the base for the development of LVIHR and triggered the expansion and popularity of this new technique. Major complications due to these materials seem minimal, but experience is still limited. Despite the increasing popularity of LVIHR, longterm consequences of intraperitoneal implantation of a synthetic mesh remain a concern. LVIHR carries a risk of adhesion formation and also has the potential to influence subsequent abdominal operations (SAOs). Reoperative findings in patients with prior LVIHR may provide the most valuable information on these issues. We, therefore, conducted a retrospective study of findings during reoperation in patients who had previously undergone an LVIHR 
using an expanded polytetrafluoroethylene mesh (DualMesh $^{\circledR}$; WL Gore, Flagstaff, AZ, USA).

\section{Patients and methods}

The medical records of 695 consecutive patients who underwent an LVIHR between January 2001 and May 2009 at our hospital were reviewed. In all patients, a DualMesh prosthesis overlapping the hernia margins by $\geq 3 \mathrm{~cm}$ was fixed with a double ring of tacks (ProTack; TycoUSS, Norwalk, CT, USA) alone $(n=385)$ or both tacks and transabdominal sutures $(n=310)$. The mean follow-up period was $35.6 \pm 20.3$ months. All patients who had SAOs were identified for this retrospective review. The study population was divided into two groups: 'early' SAOs consisting of patients who had an SAO within a few days of LVIHR either due to an early postoperative complication or the suspicion of such a complication, and 'late' SAOs consisting of patients who were devoid of early postoperative complications after their LVIHR and had an SAO at least 1 month after LVIHR. A period of 1 month was selected assuming that adhesion formation would require some time.

The following data were collected and reviewed: indication for LVIHR, mesh fixation technique, time lapse between LVIHR and SAO, indication for SAO, emergency status, and surgical approach at SAO (laparotomy or laparoscopy). Peri-operative findings reviewed included contamination level, presence and characteristics of adhesions to implants, type of procedure performed, and all problems or complications. Data acquisition included the analysis of operative reports and of videos, since nearly all relaparoscopies were recorded. At most relaparotomies, representative photographs were taken, which were also reviewed. All postoperative complications were reviewed.

The adhesions encountered were classified according to two severity scales: the first is described by Diamond [4] and the second by Zühlke et al. [5]. The first classification is not commonly used by surgeons in Europe, but it was used in the only article ever published on the issue of postoperative adhesions after LVIHR with DualMesh [6]. In the severity scale of Diamond, a score of 0 was assigned if no adhesions were present; a score of 1 if filmy, avascular adhesions were present; a score of 2 for vascular or dense adhesions, or both; and a score of 3 for cohesive adhesions. The classification of Zühlke is widely used among surgeons worldwide and reflects the characteristics of adhesiolysis. In brief, grade 0 means no adhesions; grade 1 filmy adhesions easy to take down; grade 2 when blunt dissection is sufficient; grade 3 when sharp dissection is necessary; and grade 4 when organ damage is likely during adhesiolysis.

The data were collected in an Excel database, and statistical analyses were performed using the Statistical Package for Social Sciences for Windows (SPSS Inc., Chicago, IL, USA). Statistical significance $(P<0.05)$ was determined using the $t$-test and Fisher's exact test.

\section{Results}

There were 72 (10.4\%) LVIHR patients who had SAOs for various indications. Seven (1\%) LVIHR patients had early reoperation within a few days of LVIHR. In six of them, a missed enterotomy or an infection required mesh removal. In all of these patients with peritonitis, there were no adhesions against the implant. In one patient, we decided on a 'second look' on postoperative day 3 in order to evaluate the possibility of a missed bowel injury, which was not found. The first adhesions against the mesh and tacks were present.

'Late' SAOs were performed on 65 (9.4\%) patients. There were 34 men and 31 women. Initial LVIHR was performed for an incisional hernia in 47 patients and for a primary ventral hernia in 18 patients. In 34 of these patients, the mesh was fixed both with tacks and with transabdominal sutures, and in 31 patients, the mesh was fixed only with a double ring of tacks.

The median time period between LVIHR and SAO was 14 months (range 2-67). Indications for SAOs are shown in Table 1. The two surgeons (S.R. and J.T.F.J.R.), who had also performed all LVIHRs, performed nearly all of the SAOs $(n=61 / 65$; 94\%). Surgeons without previous experience of LVIHR performed three urgent and one elective SAOs.

The vast majority of SAOs were elective $(n=62 / 65$; 95\%). Three (5\%) SAOs were urgent: one due to LVIHRunrelated bowel obstruction and two due to LVIHR-related bowel obstruction caused by an adhesion to a tack and due to a herniation through a trocar site. In the two last patients, at SAO, the mesh was removed and the hernia was closed primarily.

The initial approach during SAOs was predominantly through laparoscopy $(n=54 / 65 ; 83 \%)$. Laparotomy was performed in $11(17 \%)$ patients. None of the laparoscopies had to be converted to open surgery. During open introduction of the first trocar for an elective laparoscopic cholecystectomy by a surgeon not familiar with LVIHR, the bladder was injured in one patient. This happened because a site well below the umbilicus was chosen in an attempt to avoid the LVIHR mesh placed in the umbilical area. After repairing the injury and placing a urinary catheter, the remainder of the procedure was uneventful.

At SAO laparoscopies, the introduction of trocars through the mesh of previous LVIHR was always avoided. At SAO laparotomies, an incision through the mesh was avoided whenever possible. In four patients, this was not 
Table 1 Indications for late subsequent abdominal operations (SAOs) after laparoscopic ventral and incisional hernia repair (LVIHR) $(n=65)$

\begin{tabular}{lrr}
\hline Indication & No. & $\%$ \\
\hline LVIHR related & 42 & 65 \\
Hernia recurrence & 14 & 22 \\
Trocar site hernia & 7 & 11 \\
Symptomatic bulging & 5 & 8 \\
Chronic pain & 12 & 18 \\
Late mesh infection & 3 & 5 \\
Bowel obstruction & 1 & 1 \\
LVIHR not related & 23 & 35 \\
Gastrointestinal malignancy & 4 & 6 \\
Cholecystectomy & 3 & 5 \\
Appendicitis & 1 & 1 \\
New abdominal wall hernia & 8 & 13 \\
Paraesophageal hernia & 1 & 1 \\
Insertion of catheter for peritoneal dialysis & 2 & 3 \\
Gynecological disorder & 3 & 5 \\
Bowel obstruction & 1 & 1 \\
\hline
\end{tabular}

Table 2 Adhesion scores for late SAO after LVIHR $(n=65)$

\begin{tabular}{|c|c|c|c|}
\hline \multicolumn{2}{|c|}{ Diamond } & \multicolumn{2}{|c|}{ Zühlke } \\
\hline & $n(\%)$ & & $n(\%)$ \\
\hline 0 & $11(17)$ & 0 & $11(17)$ \\
\hline 1 & $42(65)$ & 1 & $29(44)$ \\
\hline 2 & $10(15)$ & 2 & $22(34)$ \\
\hline \multirow[t]{2}{*}{3} & $2(3)$ & 3 & $3(5)$ \\
\hline & & 4 & $0(0)$ \\
\hline
\end{tabular}

possible. In three of these patients, we decided on a prophylactic mesh removal. After completing the procedure, closure of the laparotomy included a primary closure of the hernia. Two of these three patients developed a recurrent hernia that was again repaired laparoscopically. In one patient, the mesh was closed with nonabsorbable sutures and left in place. Sixteen months later, we had to remove the mesh due to a delayed mesh infection.

Adhesions against the implanted LVIHR material were present in $83 \%(n=54 / 65)$ of patients. In $42(65 \%)$ patients, the adhesions involved omentum only, and in 12 patients (18\%), they also involved the bowel. The adhesion scores are shown in Table 2. There were no significant differences in the incidence of adhesions and their grade between the two mesh fixation techniques. There were no differences in the severity of adhesions between patients who had SAOs within 13 months of LVIHR $(n=32$; average Diamond score $=1.06$; average Zühlke score $=1.11$ ) and patients who had SAO 14 or more months after LVIHR ( $n=33$; average Diamond score $=1.13$; average Zühlke score $=1.07$ ). Adhesions were predominantly against exposed elements of LVIHR. These included either titanium tacks, especially those not completely inserted, or the edge of the mesh, which was dangling into the abdominal cavity with a partial 'rough' side of the DualMesh exposed. Even in the patient who had the 'second look' operation on the third postoperative day, the first adhesions were present at these exposed sites. No adhesions were found at the site of transabdominal sutures.

All 'late' SAOs showed a mesh completely covered with a layer of tissue resembling the patient's peritoneum. Adhesions were always against this neoperitoneum and never directly against the mesh, with the exception of those against the dangling edge of the mesh (Fig. 1). The neoperitoneum was unequivocally attached very loosely to the mesh and only more firmly against the tacks. As a consequence, adhesiolysis was, as a rule, easy and required little effort: once penetrating the neoperitoneal membrane and entering the dissection plane between the mesh and the neoperitoneum, the latter could be bluntly removed from the underlying mesh surface en bloc with all present adhesions (Fig. 2). This very efficient technique was avoided only at contaminated SAOs by using the protection of the neoperitoneum to prevent exposure of the mesh to infection. In such cases, a sharp dissection was usually needed to take adhesions down from the neoperitoneum. In a small subset of patients, a challenging adhesiolysis was necessary. This was primarily due to dense adhesions caused by extensive tacking and subsequent multiple adhesions against these tacks. We are under the impression that adhesions against tacks are, as a rule, firmer than adhesions against the neoperitoneum. No bowel or other organ injuries occurred during adhesiolysis.

The vast majority of SAOs were 'clean' procedures $(n=57 / 65 ; 88 \%)$. Eight (12\%) SAOs were contaminated:

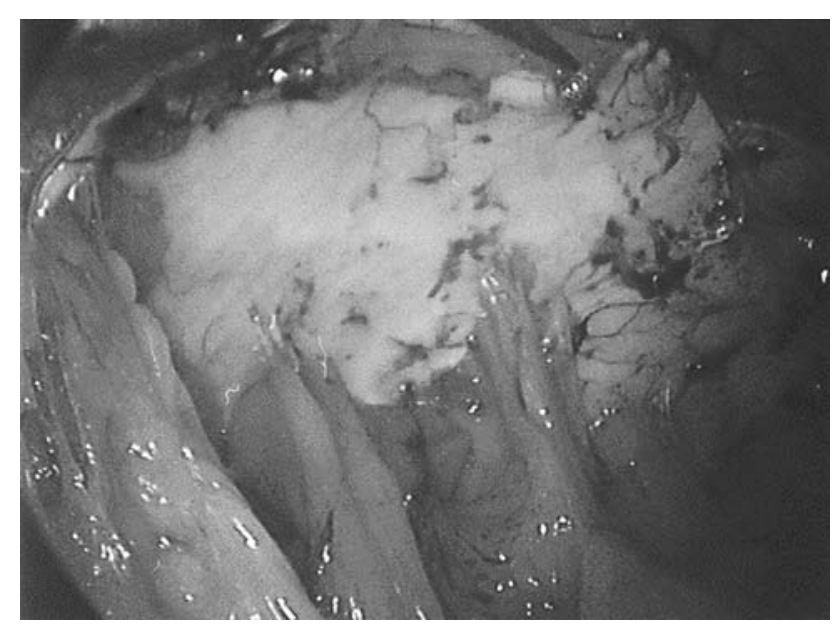

Fig. 1 Adhesions against the neoperitoneum, as seen during relaparoscopy 


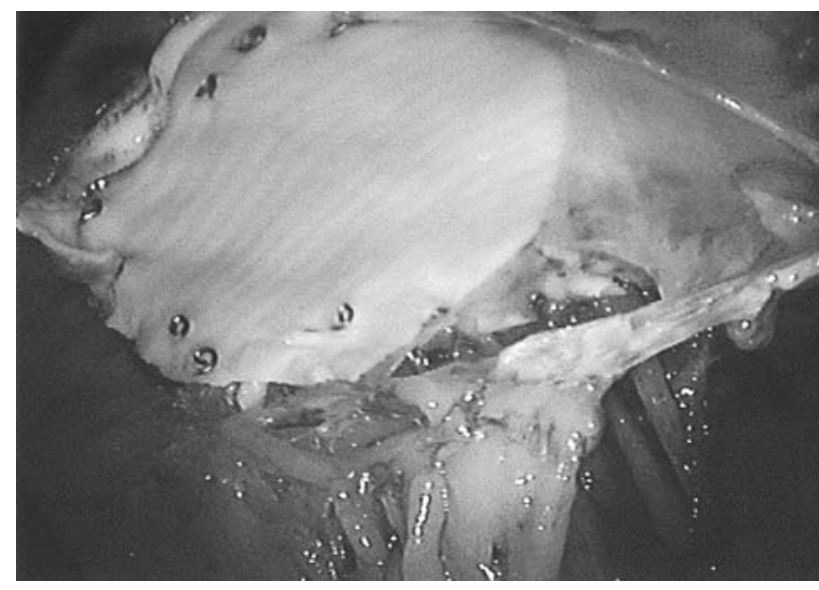

Fig. 2 Adhesiolysis during relaparoscopy by removing the neoperitoneum from the mesh

three late mesh infections required removal of the mesh, after which the hernia was closed primarily; five contaminated SAOs were not related to previous LVIHR: three colorectal resections, one perforated appendicitis, and one gallbladder empyema with peri-operative perforation of the gallbladder. In all five patients, adhesiolysis was performed with care to leave the neoperitoneum intact in order not to expose the mesh that was left in place to infection. None of these five procedures resulted in mesh infection.

With the exception of the previously mentioned bladder injury during open introduction of a trocar, there were no other peri-operative complications. SAOs were devoid of early postoperative complications. Five of the six SAO patients who underwent removal of the mesh with primary closure of the hernia defect developed a hernia recurrence as a late complication.

\section{Discussion}

Adhesion formation after LVIHR remains a concern. A large number of experimental studies addressed this important issue, but the diversity of experimental models, animals used, study times, methods used for measuring, and, above all, the extremely wide range of reported results, even for identical meshes, have made it difficult to make reliable clinical conclusions [7-12]. Very few clinical studies on adhesions after LVIHR, complications caused by them, and their impact on subsequent abdominal surgery have been published. A recent study indicated that a functional cine magnetic resonance imaging (MRI) might be valuable in the detection of adhesions against the mesh after LVIHR [13]. However, reoperative findings in patients with prior LVIHR still provide the most valuable information on the issue of adhesions after the intraperitoneal implantation of synthetic mesh. These findings, therefore, presently remain the 'gold standard' for the evaluation of the consequences of LVIHR.

Summarizing the available data, including information obtained from industry, we estimate that, so far, nearly 500,000 patients have had an LVIHR and that, each year, 100,000 or more new repairs will be performed. A certain, but still unknown, percentage of LVIHR patients will definitely undergo such surgery some time later in their life. Nearly all patients who had LVIHR at our hospital ( $n=684 / 695 ; 98.4 \%$ ) are patients who belong to the adherence area of our hospital. It can be assumed that practically all of these patients would return to our hospital for subsequent medical treatment, including SAO. Consequently, the incidence of 'late' SAOs of $9.4 \%$ that we found in this series probably realistically reflects the percentage of patients that undergo SAO within a few years of LVIHR. A similar incidence of $8.75 \%$ has been reported in the only large series on LVIHR that provided data on this issue [14].

Indication for SAO in this series was related to some complication of prior LVIHR in $65 \%$ of the patients. It is striking that in only 14 of these 42 patients had recurrence of their hernia, while the remaining 28 patients had some other complication of LVIHR. This also suggests that the recurrence rate of $2 \%(n=14 / 695)$ in this series is an insufficient parameter of importance for adverse outcomes of LVIHR. The rate of SAOs that was related to LVIHR in this series was $6 \%(n=42 / 695)$. This may provide a much more realistic rate of adverse outcome.

To the best of our knowledge, only two studies have reported on reoperative findings and adhesions in a larger number of LVIHR patients [6, 14]. One of these specifically addressed the issue of adhesions against DualMesh [6]. We found a higher incidence and more severe adhesions than what was reported in that study. Since the same material and the same technique were used in both series, the possible explanation for this difference is that we analyzed only 'late' SAOs, while the other study included a significant percentage of early reoperations. The latter are frequently performed in the presence of peritonitis that, according to our experience, seems to prevent adhesion formation. When the findings of our 'early' and 'late' SAOs are combined, our results are very similar to what has previously been published [6]. The second relevant study reported on reoperative findings after LVIHR with a Parietex mesh (Sofradim, Trevoux, France) that was fixed with transabdominal sutures only and without tacks [14]. The incidence and severity of adhesions reported in that study was lower than in this study. There are two possible explanations for this difference. The first is that DualMesh simply induces more adhesions than Parietex mesh. The second may be related to the mesh fixation method. According to our observations, a transabdominal suture was never the site of adhesions. During all SAOs that we performed, we 
were under the impression that the shape is more responsible for adhesion formation than the material. Any protruding component of LVIHR repair-either a dangling edge of the mesh or an exposed tack-is prone to becoming the site of an adhesion. Transabdominal sutures do not protrude into the abdominal cavity, which is most likely the reason for why adhesions do not develop at their sites. This observation has been mentioned in the publications of animal studies $[15,16]$.

However, as the primary endpoint, the adhesion frequency and severity might be misleading. Many patients are asymptomatic despite dense adhesions, whereas others with a single adhesion may develop small bowel obstruction [17]. This is the reason why adhesion-scoring systems remain invalidated; there is simply no useful correlation between the extent of adhesion formation and clinical outcome. In our experience, an LVIHR-related complication very rarely causes a surgical emergency. Assuming that herniation through a trocar site opening can occur after any laparoscopy, we only experienced a single case that required acute surgical intervention due to an LVIHRrelated adhesion. With a cumulative follow-up period exceeding 2,000 years for all 695 patients, this suggests the incidence of only $0.15 \%$. This may indicate that a fear of adhesions as a possible cause of long-term complications after LVIHR with a DualMesh is not justified.

When an SAO has to be performed in LVIHR patients, a surgeon with either expertise in LVIHR or at least familiarity with the technique is highly preferable. The performance of SAO after LVIHR requires detailed preoperative information on the position, size, and fixation of the previously implanted mesh. Good planning of the approach, awareness of the impact of contamination on the type of adhesiolysis, and good judgment for when to remove a mesh and when to leave it in place are all very important factors. A few problems identified in this study were related to suboptimal decisions made by surgeons without experience in LVIHR. We assume that, with an increasing number of LVIHR performed each year, the chance for such events will rise. This suggests the need for educating a wide spectrum of surgeons without experience in LVIHR on the basic principles of SAOs in LVIHR patients.

\section{Conclusion}

This study is one of the first to focus on the potential problems of performing subsequent abdominal operations (SAOs) after previous laparoscopic ventral and incisional hernia repair (LVIHR). The specific observations that: (1) not a single relaparoscopy had to be converted; (2) no inadvertent injuries were made during adhesiolysis; and (3) SAOs were practically devoid of peri- and postoperative complications, indicate that SAOs can be safely performed after LVIHR. Clinical reoperative findings on all currently used meshes for LVIHR are needed to determine the comparative effectiveness of these materials in preventing adhesions and all other complications related to LVIHR.

Open Access This article is distributed under the terms of the Creative Commons Attribution Noncommercial License which permits any noncommercial use, distribution, and reproduction in any medium, provided the original author(s) and source are credited.

\section{References}

1. Brandt CP, McHenry CR, Jacobs DG, Piotrowski JJ, Priebe PP (1995) Polypropylene mesh closure after emergency laparotomy: morbidity and outcome. Surgery 118:736-740; discussion 740741

2. Losanoff JE, Richman BW, Jones JW (2002) Entero-colocutaneous fistula: a late consequence of polypropylene mesh abdominal wall repair: case report and review of the literature. Hernia 6:144147

3. Halm JA, de Wall LL, Steyerberg EW, Jeekel J, Lange JF (2007) Intraperitoneal polypropylene mesh hernia repair complicates subsequent abdominal surgery. World J Surg 31:423-429; discussion 430

4. Diamond MP (1996) Reduction of adhesions after uterine myomectomy by Seprafilm membrane (HAL-F): a blinded, prospective, randomized, multicenter clinical study. Seprafilm Adhesion Study Group. Fertil Steril 66:904-910

5. Zühlke HV, Lorenz EM, Straub EM, Savvas V (1990) Pathophysiology and classification of adhesions (in German). Langenbecks Arch Chir Suppl II Verh Dtsch Ges Chir 1009-1016

6. Koehler RH, Begos D, Berger D, Carey S, LeBlanc K, Park A, Ramshaw B, Smoot R, Voeller G (2003) Minimal adhesions to ePTFE mesh after laparoscopic ventral incisional hernia repair: reoperative findings in 65 cases. JSLS 7:335-340

7. Dilege E, Coskun H, Gunduz B, Sakiz D, Mihmanli M (2006) Prevention of adhesion to prosthetic mesh in incisional ventral hernias: comparison of different barriers in an experimental model. Eur Surg Res 38:358-364

8. Emans PJ, Schreinemacher MH, Gijbels MJ, Beets GL, Greve JW, Koole LH, Bouvy ND (2009) Polypropylene meshes to prevent abdominal herniation. Can stable coatings prevent adhesions in the long term? Ann Biomed Eng 37:410-418

9. Harrell AG, Novitsky YW, Peindl RD, Cobb WS, Austin CE, Cristiano JA, Norton JH, Kercher KW, Heniford BT (2006) Prospective evaluation of adhesion formation and shrinkage of intra-abdominal prosthetics in a rabbit model. Am Surg 72:808813; discussion 813-814

10. Novitsky YW, Harrell AG, Cristiano JA, Paton BL, Norton HJ, Peindl RD, Kercher KW, Heniford BT (2007) Comparative evaluation of adhesion formation, strength of ingrowth, and textile properties of prosthetic meshes after long-term intra-abdominal implantation in a rabbit. J Surg Res 140:6-11

11. Schreinemacher MH, Emans PJ, Gijbels MJ, Greve JW, Beets GL, Bouvy ND (2009) Degradation of mesh coatings and intraperitoneal adhesion formation in an experimental model. Br J Surg 96:305-313

12. van't Riet M, Burger JW, Bonthuis F, Jeekel J, Bonjer HJ (2004) Prevention of adhesion formation to polypropylene mesh by collagen coating: a randomized controlled study in a rat model of ventral hernia repair. Surg Endosc 18:681-685 
13. Kirchhoff S, Ladurner R, Kirchhoff C, Mussack T, Reiser MF, Lienemann A (2009) Detection of recurrent hernia and intraabdominal adhesions following incisional hernia repair: a functional cine MRI-study. Abdom Imaging [Epub ahead of print]

14. Chelala E, Thoma M, Tatete B, Lemye AC, Dessily M, Alle JL (2007) The suturing concept for laparoscopic mesh fixation in venatral and incisional hernia repair: mid-term analysis of 400 cases. Surg Endosc 21:391-395

15. Karahasanoglu T, Onur E, Baca B, Hamzaoglu I, Pekmezci S, Boler DE, Kilic N, Altug T (2004) Spiral tacks may contribute to intra-abdominal adhesion formation. Surg Today 34:860864

16. LeBlanc KA, Stout RW, Kearney MT, Paulson DB (2003) Comparison of adhesion formation associated with Pro-Tack (US Surgical) versus a new mesh fixation device, Salute (ONUX Medical). Surg Endosc 17:1409-1417

17. Harris DA, Topley N (2008) Peritoneal adhesions. Br J Surg 95:271-272 